\title{
METRIZABLE SPACES WHERE THE INDUCTIVE DIMENSIONS DISAGREE
}

\author{
JOHN KULESZA
}

\begin{abstract}
A method for constructing zero-dimensional metrizable spaces is given. Using generalizations of Roy's technique, these spaces can often be shown to have positive large inductive dimension. Examples of $\mathbf{N}$-compact, complete metrizable spaces with ind $=0$ and Ind $=1$ are provided, answering questions of Mrowka and Roy. An example with weight $c$ and positive Ind such that subspaces with smaller weight have Ind $=0$ is produced in ZFC. Assuming an additional axiom, for each cardinal $\lambda$ a space of positive Ind with all subspaces with weight less than $\lambda$ strongly zero-dimensional is constructed.
\end{abstract}

\section{INTRODUCTION}

There are three classical notions of dimension for a normal topological space $X$. The small inductive dimension, denoted $\operatorname{ind}(X)$, is defined by $\operatorname{ind}(X)=-1$ if $X$ is empty, $\operatorname{ind}(X) \leq n$ if each $p \in X$ has a neighborhood base of open sets whose boundaries have ind $\leq n-1$, and $\operatorname{ind}(X)=n$ if $\operatorname{ind}(X) \leq n$ and $n$ is as small as possible. The large inductive dimension, denoted $\operatorname{Ind}(X)$, is defined by $\operatorname{Ind}(X)=-1$ if $X$ is empty, $\operatorname{Ind}(X) \leq n$ if each closed $C \subseteq X$ has a neighborhood base of open sets whose boundaries have Ind $\leq n-1$, and $\operatorname{Ind}(X)=n$ if $\operatorname{Ind}(X) \leq n$ and $n$ is as small as possible. The covering dimension, denoted by $\operatorname{dim}(X)$, is defined by $\operatorname{dim}(X)=-1$ if $X$ is empty, $\operatorname{dim}(X) \leq n$ if for each finite open cover of $X$ there is a finite open refining cover such that no point is in more than $n+1$ of the sets in the refining cover and, $\operatorname{dim}(X)=n$ if $\operatorname{dim}(X) \leq n$ and $n$ is as small as possible. It is well known that for separable metric spaces, all three dimensions coincide and that for arbitrary metric spaces Ind = dim; however, it is possible to have ind $<$ Ind, and we will call a metric space with this property a gap metric space. Very few examples of gap metric spaces are in the literature. The original example is Roy's space $\Delta$, described in 1962 (see [R1, R2]). Nyikos showed that the Engelking-Mrowka conjecture is false in [NY] by showing that $\Delta$ is not $\mathbf{N}$-compact, that is, that $\Delta$ is not homeomorphic to a closed subspace of $\mathbf{N}^{x}$ for any cardinal $\alpha$. Later Mrowka produced an $\mathbf{N}$-compact gap metric space, which he called $\mu_{0}$, in [M]. Most recently, examples with weight equal to $\omega_{1}$

Received by the editors March 24, 1988 and, in revised form, August 10, 1988. 1980 Mathematics Subject Classification (1985 Revision). Primary 54F45, 54G99. Key words and phrases. Metrizable space, full set, dimension. 
were produced independently by Ostaszewski in [O] and by the author in [K1].

All of the examples mentioned above as well as those constructed here satisfy ind $=0$, that is they have bases of clopen sets, sets which are simultaneously closed and open; such spaces are called zero-dimensional. We are concerned with metrizable spaces where ind $=0$ and one (hence both) of the other dimensions are not. (They will be 1.) It is apparent from the definition that $\operatorname{Ind}(X)=0$ if and only if whenever $F_{1}$ and $F_{2}$ are disjoint closed subsets of $X$, there is a clopen set containing $F_{1}$ which is disjoint from $F_{2}$. Thus our approach to showing Ind $>0$ will be to exhibit a pair of disjoint closed sets for which no such clopen set exists. We will call a metric space for which Ind $=0$ strongly zero-dimensional, although this term refers to another dimension (Dim, not discussed here) being zero; there is no confusion because $\mathrm{Dim}=$ Ind for metric spaces.

In this paper a rather general method for producing gap metric spaces is described; the method involves using well-chosen subspaces of countably infinite products of well-chosen factor spaces. The advantages of this technique are that the examples are, in general, much simpler than existing examples, and the properties can be varied to a large extent. Furthermore, these spaces are fairly common. For instance, the example in [K1] (the first application of this method) is a subspace of $\omega_{1}^{\omega}$, and one of the examples in this paper is a subspace of $M^{\omega}$ ( $M$ denotes the Michael line, the space whose point set is the real numbers and whose topology is obtained by taking sets of the form $O \cup P$ as open, where $O$ is a usual open set and $P$ is a subset of the irrationals). Finally, the product space gives a reasonable setting in which to look for spaces where the discrepancy in dimension is greater than 1.

In $\S I I$ general notations and key background lemmas are given. §III gives the method for producing zero-dimensional metrizable spaces; theorems are proved which show the spaces are in fact zero-dimensional, and a further theorem gives conditions which guarantee completeness.

In $\S I V$ we apply the method of $\S I I I$ to produce two $\mathbf{N}$-compact examples of gap metric spaces. Both are complete, and thus affirmatively answer the question of Mrowka, "Do there exist complete $\mathbf{N}$-compact metric spaces with dim > 0 ?", posed in $[A]$. The first space is simple to describe. The second, additionally, answers a question of Roy's. He had asked if non-N-compactness was a property of all subspaces of $\Delta$ with positive Ind. As the second example embeds in $\Delta$ (the technical proof of which is in [K2]), it gives a negative answer to Roy's question. The second example is best understood as a modification of the first.

Since the discovery of gap metric spaces of weight $\omega_{1}$ (the weight of a topological space is the minimal cardinality of a base for the topology), an obvious question becomes "Does every gap metric space contain a gap metric subspace of weight $\omega_{1}$ ?" or, more generally, "Is there a cardinal $\lambda$ such that every gap metric space contains a gap metric subspace of weight $\leq \lambda$ ?". It is not too hard, 
using the ideas in [O], to show $\Delta$ contains a weight $\omega_{1}$ gap metric subspace; for $\mu_{0}$ the answer is not clear. In $\S \mathrm{V}$, a gap metric space of weight $\mathfrak{c}$ is described with the property that every subspace of weight less that $c$ is strongly zero-dimensional; thus the first question has a negative answer assuming the negation of $\mathrm{CH}$. In $\S \mathrm{VI}$, assuming a set theoretic axiom $E^{*}$, it is shown that, for any cardinal $\lambda$, there is a gap metric space such that any subspace of cardinality less than $\lambda$ has Ind $=0$, giving a negative answer to the second question. The examples of this section seem to be the first examples of nonstrongly zerodimensional metrizable spaces with all small (where small is as large as desired) subspaces strongly zero-dimensional. Furthermore, in the examples, closed sets of cardinality less than $\lambda$ have neighborhood bases of clopen sets; the closed sets which exhibit Ind $>0$ have cardinality at least $\lambda$.

With regard to the methods of this paper, they should be viewed as simplifications and generalizations of methods employed by Roy and later by Mrowka.

The author gratefully acknowledges many valuable suggestions and comments by the referee and by Professor Ronnie Levy.

\section{Preliminaries}

For a set $I$ and a collection $\left\{X_{i}: i \in I\right\}$ of sets, $\prod_{i \in I} X_{i}$ denotes $\{f: f$ is a function, $\operatorname{domain}(f)=I$, and $\left.\forall i \in I, f(i) \in X_{i}\right\}$; correspondingly, $X^{I}$ will denote $\{\sigma$ : domain $(\sigma)=I$, and $\operatorname{image}(\sigma) \subseteq X\}$. If $I \in \omega+1$ and domain $(\sigma)=I$, then $\sigma$ is called a sequence. If $\sigma$ is a sequence and $D$ is a collection of sequences, then $|\sigma|$ denotes domain $(\sigma)$ and

$$
|D|=\sup \{|\tau|: \tau \in D\} ;
$$

also $\left.\sigma\right|_{n}$ and $\left.D\right|_{n}$ denote the appropriate restrictions to $n$. We do not distinguish between $\sigma$ and the ordered $|\sigma|$-tuple $(\sigma(0), \sigma(1), \sigma(2), \ldots)$.

We adopt the following notation which is useful in manipulating sequences. Let $\left\{X_{i}: i \in \omega\right\}$ be a given collection of sets, let $\sigma, \tau$ be sequences, and let $D, E$ be collections of sequences such that $\gamma(i) \in X_{i}$ for $\gamma \in\{\sigma, \tau\} \cup D \cup E$ and $i<|\gamma|$, and such that $|\sigma|<\omega$ and $|D|<\omega$. We define

$$
\langle\sigma, \tau\rangle=(\sigma(0), \sigma(1), \ldots, \sigma(|\sigma|), \tau(0), \tau(1), \ldots),
$$

that is $\langle\sigma ; \tau\rangle$ is the concatenation of $\sigma$ and $\tau$.

$$
\langle\sigma ; E\rangle=\{\langle\sigma, \varepsilon\rangle: \varepsilon \in E\}, \quad\langle D ; E\rangle=\{\langle\delta, \varepsilon\rangle: \delta \in D, \varepsilon \in E\},
$$

and

$$
\overline{\langle\sigma ; D\rangle}=\left\{\psi \in \prod X_{i}: \text { there is } n \in \omega \text { such that }\left.\psi\right|_{n} \in\langle\sigma ; D\rangle\right\} .
$$

Later on, when we are discussing a special subset $R$ of $\prod X_{i},\langle\overline{\sigma ; D}\rangle$ will instead be used (ambiguously) to mean $\langle\overline{\sigma ; D}\rangle \cap R$. If $P$ is a property of subsets of $X$, then $P$ is countably coadditive on $X$ means that, if $X=\bigcup_{i \in \omega} X_{i}$, then one of the $X_{i}$ 's has property $P$. Most notably for our purposes, if $X$ is an uncountable set then the property $U$ of being uncountable is countably 
coadditive on $X$; also if $X$ is a second category in its own topological space, then the property $S$ of being second category in $X$ is countably coadditive.

The following definition and lemmas are used to show that the examples constructed have positive Ind. Special cases of Lemmas 3 and 4 were known to Roy [R2]; these lemmas were discovered independently by Fleissner [F]; Lemmas 3, 4 and 5 were used by Mrowka in [M] in the language of ramified products.

1. Definition. Let $\left\{X_{i}: i \in I\right\}$, where $I \in \omega+1$, be a collection of sets such that property $P$ is countably coadditive on each $X_{i}$. Then $Y \subseteq \prod X_{i}$ is a $P$-full subset of $\Pi X_{i}$ iff for each $\sigma \in Y$ and $k \in I$, the subset $\left\{\tau(k):\left.\tau\right|_{k-1}=\left.\sigma\right|_{k-1}\right\}$ of $X_{k}$ has property $P$ on $X_{k}$.

As remarked above, we will be especially concerned with $U$-full and $S$-full subsets.

2. Definition. Let $\left\{X_{i}: i \in I\right\}$, where $I \in \omega+1$, be a collection of second countable spaces. Then $Y \subseteq \prod X_{i}$ is a super $S$-full subset of $\prod X_{i}$ iff for each $\sigma \in Y$ and $k \in I,\left\{\tau(k):\left.\tau\right|_{k-1}=\left.\sigma\right|_{k-1}\right\}$ is second category everywhere on $X_{k}$.

3. Lemma. Let $\left\{X_{i}: i<n\right\}$ where $n \in \omega$, be a collection of sets with property $P$ countably coadditive on each $X_{i}$, and let $Y \subseteq \prod X_{i}$ be a $P$-full subset of $\prod X_{i}$. Then, if $Y=\bigcup_{i \in \omega} Y_{i}$, there is a $j \in \omega$ such that $Y_{j}$ contains a P-full subset of $\prod X_{i}$.

4. Lemma. Let $\left\{X_{i}: i \in \omega\right\}$ be a collection of sets with property $P$ countably coadditive on each $X_{i}$; let $X=\prod X_{i}$ and let $\left\{C_{i}: i \in \omega\right\}$ be a collection of subsets of $X$ satisfying:

(i) $C_{i} \subseteq C_{i+1} \quad \forall i \in \omega$,

(ii) $X=\bigcup C_{i}$, and

(iii) if $\sigma \in C_{n}$, then $\left\{\tau \in X:\left.\tau\right|_{n}=\left.\sigma\right|_{n}\right\} \subseteq C_{n}$.

Then there is a $k$ such that $\left.C_{k}\right|_{k}$ contains a $P$-full subset of $\prod_{i<k} X_{i}$.

5. Lemma. Let $\left\{X_{i}: i<n\right\}$ be a collection of second countable spaces, and let $K$ be an $S$-full subset of $\Pi X_{i}$; there is a $K^{\prime} \subseteq K$ and an $O_{i} \subseteq X_{i}$ for each $i<n$, where $O_{i}$ is a nonempty open subset of $X_{i}$ such that $K^{\prime}$ is a super $S$-full subset of $\Pi O_{i}$.

If $X$ is a topological space, then $Y \subseteq X$ is a Bernstein subset of $X$ provided $Y \cap K \neq \varnothing$ and $(X \backslash Y) \cap K \neq \varnothing$ if $K$ is an uncountable closed subset of $X$. If $X$ is a complete, separable metric space with no isolated points then $X$ contains a Bernstein set, and each Bernstein subset of $X$ is second category everywhere on $X$. Furthermore, for such $X$, if $O$ is an open cover in $X$ of a Bernstein subset of $X$, then $X \backslash(\cup O)$ is countable. 


\section{A METHOD FOR CONSTRUCTING ZERO DIMENSIONAL}

\section{METRIZABLE SPACES}

1. The spaces will be subspaces specified in Definition 1.3 where the factor spaces are always of the form given in Definition 1.1. Theorem 1.4 gives the main result, and Theorem 1.6 also gives conditions which guarantee completeness of a space satisfying the hypotheses of 1.4. The rest of the section is devoted to proving Theorem 1.4 and 1.6.

1.1. Definition. A triple $(X, A, B)$ is a tame triple provided $X$ is a first countable, $T_{1}$ topological space, $X=A \cup B$ where $A \cap B=\varnothing$, and the elements of $A$ are isolated in $X$.

1.2. Examples of the tame triples. (i) $\left(E_{\lambda}, S, L\right)$, where $\lambda$ is an ordinal with the order topology, $S$ is the set of successor ordinals in $\lambda, L$ is the set of limit ordinals in $\lambda$ with countable cofinality, and $E_{\lambda}=S \cup L$.

(ii) $(M, \mathbf{I}, \mathbf{Q})$, where $M$ denotes the Michael line, I the irrational points and $\mathbf{Q}$ the rational points.

(iii) $(A(Y), N, O)$, where $A(Y)$ denotes the Alexandroff duplicate of the (first countable) space $Y, O$ represents the "old" points, and $N$ the "new" points.

1.3. Definition. Let $\left\{\left(X_{i}, A_{i}, B_{i}\right): i \in \omega\right\}$ be a collection of tame triples. A set $X \subseteq \prod X_{i}$ respects $\left\{\left(X_{i}, A_{i}, B_{i}\right): i \in \omega\right\}$ provided $X$ satisfies the following:

(i) $X=P_{1} \cup P_{2}^{0} \cup P_{2}^{1} \cup P_{2}^{2} \cup \cdots$, where $P_{1}=X \cap\left(\prod_{i \in \omega} A_{i}\right)$, and for $k \in \omega$,

$$
P_{2}^{k}=X \cap\left(\left(\prod_{i<k} A_{i}\right) \times B_{k} \times\left(\prod_{i>k} A_{i}\right)\right) .
$$

(ii) There is a function $\phi_{1}: \omega \rightarrow \omega$ satisfying, for each $k \in \omega$ :

(a) if $b_{1}, b_{2} \in B_{k}$, and $b_{1} \neq b_{2}$, then

$$
\pi_{\phi_{1}(k)}\left(X \cap \pi_{k}^{-1}\left(b_{1}\right)\right) \cap \pi_{\phi_{1}(k)}\left(X \cap \pi_{k}^{-1}\left(b_{2}\right)\right)=\varnothing,
$$

and

(b) $\phi_{1}(k)>k$.

(iii) There is a function $\phi_{2}: \omega^{2} \rightarrow \omega$ satisfying for each $k_{1}, k_{2} \in \omega$, where $k_{1} \neq k_{2}$ :

(a) $\pi_{\phi_{2}\left(k_{1}, k_{2}\right)}\left(P_{2}^{k_{1}}\right) \cap \pi_{\phi_{2}\left(k_{1}, k_{2}\right)}\left(P_{2}^{k_{2}}\right)=\varnothing$, and

(b) $\phi_{2}\left(k_{1}, k_{2}\right)>\max \left\{k_{1}, k_{2}\right\}$.

The following is the main theorem for verifying that examples are metrizable.

1.4. Theorem. Let $\left\{\left(X_{i}, A_{i}, B_{i}\right): i \in \omega\right\}$ be a collection of tame triples and let $X \subseteq \prod X_{i}$ be a subspace which respects $\left\{\left(X_{i}, A_{i}, B_{i}\right): i \in \omega\right\}$. Then:

(i) $X$ is zero-dimensional,

(ii) $X$ is metrizable, and

(iii) $\operatorname{Ind}(X) \leq 1$. 
We remark that it is not necessary that the factor spaces themselves be zerodimensional. The proof of this theorem is postponed until subsection 3 . We continue giving conditions which guarantee completeness.

1.5. Definition. Let $\left\{X_{i}: i \in \omega\right\}$ be a collection of sets. A set $X \subseteq \prod X_{i}$ is an approximately complete subset of $\prod X_{i}$ provided: if $\sigma \in \prod X_{i}$ and $\{\tau \in$ $\left.X:\left.\tau\right|_{k}=\left.\sigma\right|_{k}\right\} \neq \varnothing$ for each $k \in \omega$, then $\sigma \in X$.

We remark that the approximately complete subsets are precisely those which are closed in the product $\Pi\left(X_{i}, D_{i}\right)$ where, for each $i, D_{i}$ is the discrete topology on $X_{i}$.

1.6. Theorem. If $\left\{\left(X_{i}, A_{i}, B_{i}\right): i \in \omega\right\}$ is a collection of tame triples, $X \subseteq \prod X_{i}$ respects $\left\{\left(X_{i}, A_{i}, B_{i}\right): i \in \omega\right\}$ and is an approximately complete subset of $\prod X_{i}$, then $X$ is completely metrizable.

2. Theorem 1.6 will be proved in subsection 4.2. We now introduce some notation, which will be standard, and prove lemmas which will be necessary for proving 1.4 and 1.6. Throughout we will assume a fixed collection $\left\{\left(X_{i}, A_{i}, B_{i}\right): i \in\right.$ $\omega\}$ of tame triples and a fixed $X \subseteq \prod X_{i}$ which respects $\left\{\left(X_{i}, A_{i}, B_{i}\right): i \in \omega\right\}$.

2.1. For each $i \in \omega$, and each $p \in B_{i}$, let $\left\{N_{n}^{i}(p): n \in \omega\right\}$ be a nested local base at $p$ in $X_{i}$.

2.2. For each $\sigma \in X$, we define a nested local base $\left\{V_{i}(\sigma): i \in \omega\right\}$ as follows:

(i) if $\sigma \in P_{1}$, then $V_{i}(\sigma)=\left\{\tau \in X:\left.\tau\right|_{i}=\left.\sigma\right|_{i}\right\}$ for $i \in \omega$,

(ii) if $\sigma \in P_{2}^{k}$ and $i>k$, then $V_{i}(\sigma)=\left\{\tau \in X:\left.\tau\right|_{k}=\left.\sigma\right|_{k}, \tau(k) \in\right.$ $N_{i}^{k}(\sigma(k))$, and for $\left.k<j<i, \tau(j)=\sigma(j)\right\}$. For $i \leq k$ let $V_{i}(\sigma)=$ $V_{k+1}(\sigma)$.

It is easy to check that for $\sigma \in X,\left\{V_{i}(\sigma): i \in \omega\right\}$ is a nested local base at $\sigma$ in the subspace topology on $X$ (as a subset of $\prod X_{i}$ ).

2.3. For $i \in \omega$, let $G_{i}=\left\{V_{i}(\sigma): \sigma \in X\right\}$. It is clear that $G_{i}$ covers $X, G_{i+1}$ refines $G_{i}$, and $G=\bigcup\left\{G_{i}: i \in \omega\right\}$ is a base for $X$.

We now prove lemmas which establish relationship among the various basis elements we have just described.

2.4. Lemma. For each $k \in \omega, n>\max \left\{k+1, \phi_{1}(k)\right\}$, and $\sigma, \tau \in P_{2}^{k}$, either $V_{n}(\sigma)=V_{n}(\tau)$ or $V_{n}(\sigma) \cap V_{n}(\tau)=\varnothing$.

Proof. Either $\left.\sigma\right|_{n}=\left.\tau\right|_{n}$ in which case $V_{n}(\sigma)=V_{n}(\tau)$, or else there is a $j<n$ such that $\sigma(j) \neq \tau(j)$. If $j \neq k$ then obviously $V_{n}(\sigma) \cap V_{n}(\tau)=\varnothing$. If $j=k$, then $\sigma\left(\phi_{1}(k)\right) \neq \tau\left(\phi_{1}(k)\right)$ and $V_{n}(\sigma) \cap V_{n}(\tau)=\varnothing$.

2.5. Lemma. If $\sigma \in P_{2}^{k_{1}}$ and $\tau \in P_{2}^{k_{2}}$, where $k_{1} \neq k_{2}$, then $V_{n}(\sigma) \cap V_{n}(\tau)=\varnothing$ for $n>\phi_{2}\left(k_{1}, k_{2}\right)$.

Proof. $V_{n}(\sigma) \subseteq\left\{\gamma \in X: \gamma\left(\phi_{2}\left(k_{1}, k_{2}\right)\right)=\sigma\left(\phi_{2}\left(k_{1}, k_{2}\right)\right)\right\}$ and $V_{n}(\tau) \subseteq\{\gamma \in$ $\left.X: \gamma\left(\phi_{2}\left(k_{1}, k_{2}\right)\right)=\tau\left(\phi_{2}\left(k_{1}, k_{2}\right)\right)\right\}$. By assumption the two sets do not intersect. 
2.6. Lemma. If $\sigma \in P_{1}$ and $k \in \omega$, then there is $t \in \omega$ such that $V_{t}(\sigma) \cap V_{t}(\tau)=$ $\varnothing$ whenever $\tau \in P_{2}^{k}$.

Proof. Let $t_{1}=\phi(k)+1$. If $\tau \in P_{2}^{k}$ and $V_{t_{1}}(\sigma) \cap V_{t_{1}}(\tau) \neq \varnothing$, then, since $\tau(k)$ is determined by $\sigma\left(\phi_{1}(k)\right)$, there is a neighborhood $N_{m}^{k}(\tau(k))$ in $X_{k}$ which does not contain $\sigma(k)$, since $\sigma(k) \in A_{k}$ and $\tau(k) \in B_{k}$. But then $t=\max \left\{t_{1}, m\right\}$ suffices for the lemma.

2.7. Lemma. If $\sigma \in P_{1}$ and $m \in \omega$, then there is $t \in \omega$ such that $V_{t}(\sigma) \cap V_{t}(\tau)=$ $\varnothing$ for all $\tau \in P_{2}^{i}$ where $i<m$.

Proof. Apply Lemma $2.6 \mathrm{~m}$ times.

2.8. Lemma. If $m \in \omega, \sigma \in P_{1} \cup \bigcup_{j<m} P_{2}^{j}$ and $\tau \notin P_{2}^{i}$ for $i \leq m$, then either $V_{t}(\tau) \subseteq V_{m}(\sigma) \forall t>m$, or else $V_{t}(\tau) \cap V_{m}(\sigma)=\varnothing \quad \forall t>m$.

Proof. If $\tau \in V_{m}(\sigma)$, then $\left\{\gamma:\left.\gamma\right|_{m+1}=\left.\tau\right|_{m+1}\right\} \subseteq V_{m}(\sigma)$; hence $V_{t}(\tau) \subseteq V_{m}(\sigma)$ for all $t>m$, as $V_{m}(\tau) \subseteq V_{m}(\sigma)$. On the other hand, $\tau \notin V_{m}(\sigma)$ implies $V_{m}(\tau) \cap V_{m}(\sigma)=\varnothing$, thus $V_{t}(\tau) \cap V_{m}(\sigma)=\varnothing$ for $t>m$.

3. Proof of Theorem 1.4. First we show

\section{1. $X$ is zero-dimensional.}

This will follow immediately from 3.1.1 and 3.1.2.

3.1.1. If $\sigma \in P_{1}$ and $i \in \omega$, then $V_{i}(\sigma)$ is a clopen set in $X$.

Proof. $\tau \in V_{i}(\sigma)$ iff $\left.\tau\right|_{i}=\left.\sigma\right|_{i}$. If $\tau(j) \neq \sigma(j)$ for some $j<i$, then either $\tau(j) \in A_{j}$, in which case $V_{i}(\tau) \cap V_{i}(\sigma)=\varnothing$, or else $\tau(j) \in B_{j}$, in which case there is $N_{m}^{j}(\tau(j))$ which does not contain $\sigma(j)$. Thus for $r \geq \max \{i, m\}$, $V_{r}(\tau) \cap V_{i}(\sigma)=\varnothing$. Hence $V_{i}(\sigma)$ is clopen.

3.1.2. If $\sigma \in P_{2}^{k}$ and $i>\max \left\{k+1, \phi_{1}(k)\right\}$, then $V_{i}(\sigma)$ is clopen.

Proof. Suppose $\tau$ is in the closure of $V_{i}(\sigma)$. If $\tau \in P_{2}^{k}$, then by Lemma 2.4, $\tau \in V_{i}(\sigma)$. Clearly if $\tau \in P_{1}, \tau \in V_{i}(\sigma)$. If $\tau \in P_{2}^{j}$, where $i<j$, then Lemma 2.8 gives that $\tau \in V_{i}(\sigma)$. Thus we only need to consider $\tau \in P_{2}^{j}$, where $j \leq i$ and $j \neq k$. Here an argument similar to that in 3.1.1 works.

3.2. $X$ is metrizable. We make use of the following theorem due to Frink (see [N, p. 33]).

3.2.1. Theorem. $A T_{2}$ space $X$ is metrizable if there exists a countable open neighborhood base $\left\{V_{i}(\sigma): i \in \omega\right\}$ for each $\sigma \in X$ satisfying: For each $\sigma \in X$ and $i \in \omega$ there is a $t \in \omega$ such that $V_{t}(\sigma) \cap V_{t}(\tau) \neq \varnothing$ implies $V_{t}(\tau) \subseteq V_{i}(\sigma)$.

We will show that the sets $\left\{V_{i}(\sigma): i \in \omega\right\}$ for $\sigma \in X$ satisfy the condition of Theorem 3.2.1. Assume $i \in \omega$ and $\sigma \in X$ are given.

3.2.2. The condition of Theorem 3.2.1 is met if $\sigma \in P_{1}$. 
Proof. If $\tau \in P_{2}^{j}$ where $j>i$, then Lemma 2.8 guarantees that for any $s>i$ either $V_{s}(\tau) \subseteq V_{s}(\sigma)$ or $V_{s}(\tau) \cap V_{i}(\sigma)=\varnothing$. In case $\tau \in P_{1}$ this is also obviously true. By Lemma 2.7 we can find $u \in \omega$ such that $V_{u}(\sigma) \cap V_{u}(\tau)=\varnothing$ for any $\tau \in P_{2}^{j}$ where $j \leq i$. Thus we may choose $t=\max \{u, i+1\}$.

3.2.3. The condition of Theorem 3.2.1 is met for $\sigma \in P_{2}^{k}$.

Proof. For $\tau \in P_{1} \cup\left(\bigcup_{j<i} P_{2}^{j}\right)$, as in 3.2.2, for any $s>i, V_{s}(\tau) \subseteq V_{i}(\sigma)$ or $V_{s}(\tau) \cap V_{i}(\sigma)=\varnothing$. Choose $u=\max \left\{\phi_{2}(k, j): j \leq i\right\}$. Then $V_{u}(\tau) \cap V_{u}(\sigma)=\varnothing$ for $\tau \in P_{2}^{i}, i \leq m$. Thus let $t=\max \{u, i+1\}$.

From 3.2.2 and 3.2.3 we conclude, via Frink's Theorem, that $X$ is metrizable. It remains to show:

3.3. $\operatorname{Ind}(X) \leq 1$. We will show for each $k \in \omega$, that $\operatorname{Ind}\left(P_{2}^{k}\right)=0$, that $P_{2}^{k}$ is closed in $X$, and that $\operatorname{Ind}\left(P_{1}\right)=0$. It follows from [NA, Theorem 9-10] that $\operatorname{Ind}\left(\bigcup_{k \in \omega} P_{2}^{k}\right)=0$, and so $X$ is the metrizable union of two subspaces $P_{1}$ and $\bigcup_{k \in \omega} P_{2}^{k}$ which have Ind $=0$. By [NA, Theorem 12-6], $\operatorname{Ind}(X) \leq 1$.

3.3.1. For $k \in \omega, \operatorname{Ind}\left(P_{2}^{k}\right)=0$.

Proof. Lemma 2.4 gives that, for $n \geq \phi_{1}(k)$, the collection $O_{n}^{k}=\left\{V_{n}(\sigma): \sigma \in\right.$ $\left.P_{2}^{k}\right\}$ is an order one cover of $P_{2}^{k}$. But $O_{n+1}^{k}$ refines $O_{n}^{k}$, and $\bigcup_{j \in \omega}^{n} O_{j}^{k}$ is a base for $P_{2}^{k}$. Hence by [NA, Theorem 12-6], $\operatorname{Ind}\left(P_{2}^{k}\right)=0$.

3.3.2. $P_{2}^{k}$ is a closed set in $X$.

Proof. This follows immediately from Lemmas 2.5 and 2.6.

3.3.3. $\operatorname{Ind}\left(P_{1}\right)=0$.

Proof. Let $O_{n}=\left\{V_{n}(\sigma): \sigma \in P_{1}\right\}$ and proceed as in 3.3.1.

Thus we have shown $\operatorname{Ind}(X) \leq 1$, and the proof of Theorem 1.4 is complete.

4. We prove Theorem 1.6 , so we assume $X$ is additionally approximately complete. We only need to show (e.g., see [N, 3.5-3.8]):

4.1. $\left\{G_{i}: i \in \omega\right\}$ is a complete sequence of open covers for $X$, that is, if $\left\{g_{i}: i \in \omega\right\}$ is a collection of sets in $G$ such that $g_{i} \in G_{i}$ for all $i \in \omega$ and $\left\{g_{i}: i \in \omega\right\}$ has the finite intersection property, then $\bigcap_{i \in \omega} g_{i} \neq \varnothing$.

We will use, without proof, the following easy reduction of 4.1 (use e.g. Theorem 3.2.1).

4.2. Lemma. If for $\left\{g_{i}\right\}$ as in 4.1 there is a subsequence $\left\{\eta_{i}: i \in \omega\right\}$ of $\omega$ such that $\bigcap_{i \in \omega} g_{\eta_{i}} \neq \varnothing$, then $\bigcap_{i \in \omega} g_{i} \neq \varnothing$.

Theorem 1.6 is proved by considering three cases. Assume $\left\{g_{i}\right\}$ as in 4.1; for each $i \in \omega$ choose $\sigma_{i} \in X$ such that $g_{i}=V_{i}\left(\sigma_{i}\right)$. 
Case 1. There is an increasing sequence $\left\{\eta_{i}: i \in \omega\right\}$ with $\sigma_{\eta_{i}} \in P_{1}$. Since no finite intersection of the $g_{i}$ 's is empty, then for $i, j>t,\left.\sigma_{\eta_{i}}\right|_{t}=\left.\sigma_{\eta_{j}}\right|_{t}$. Setting $\tau=\left(\sigma_{\eta_{1}}(0), \sigma_{\eta_{2}}(1), \sigma_{\eta_{3}}(2), \ldots\right)$, approximate completeness guarantees that $\tau \in X$; furthermore it is clear that $\tau \in \bigcap_{i \in \omega} g_{\eta_{i}}$, hence by Lemma 4.2, $\bigcap_{i \in \omega} g_{i} \neq \varnothing$.

Case 2. There is a fixed $k \in \omega$ and an increasing sequence $\left\{\eta_{i}: i \in \omega\right\}$ with $\sigma_{\eta_{i}} \in P_{2}^{k}$. We may assume $\eta_{0}>k$; as in Case $1, \tau=\left(\sigma_{\eta_{1}}(0), \sigma_{\eta_{2}}(1), \ldots\right) \in X$ by approximate completeness, and $\tau \in \bigcap_{i \in \omega} g_{i}$.

Case 3. There is an increasing sequence $\left\{\eta_{i}: i \in \omega\right\}$ with $\sigma_{\eta_{i}} \in P_{2}^{k_{\eta_{i}}}$ where $\left\{k_{\eta_{i}}: i \in \omega\right\}$ is also increasing with $i$. Thus for $\eta_{i}, \eta_{y}>t,\left.\sigma_{\eta_{i}}\right|_{t}=\left.\sigma_{\eta_{i}}\right|_{t}=\left.\tau\right|_{t}$ where $\tau=\left(\sigma_{\eta_{1}}(0), \sigma_{\eta_{2}}(1), \ldots\right)$. Again we get $\tau \in \bigcap_{i \in \omega} g_{i}$.

Since one of the three cases must occur, the conclusion of 4.1 must follow.

\section{Applications to N-compact spaces}

The letter $C$ will denote both the point set $\{0,1\}^{\omega}$ and the set with the usual Cantor set topology. For $x \in C, J_{n}(x)$ denotes the usual basic neighborhood of $x$ in $C$ given by $\left\{y \in C:\left.y\right|_{n}=\left.x\right|_{n}\right\}$. End denotes the set of end points of $C$ (those which take on either 0 or 1 only finitely many times), and Int denotes the set of interior points $\left(C \backslash\right.$ End). By $M_{C}$, we mean the topological space with point set $C$ and a base given by sets $\{\{x\}: x \in \operatorname{Int}\} \cup\left\{J_{n}(x): x \in\right.$ End $\}$. Thus $M_{C}$ is the "Michael Cantor set"; we use $M_{C}$ instead of $M$ purely for technical reasons.

1. Example E1. Let $S=\{$ End $\} \cup\left\{A_{i, j}: i, j \in \omega\right\}$ be a partition of $C$ such that the $A_{i, j}$ are second category everywhere subsets of $C$ (Bernstein subsets will do), $\forall i, j \in \omega$. Let $\left\{e_{i}: i \in \omega\right\}$ be an enumeration of End. Then $\mathrm{E} 1=$ $P_{1} \cup\left(\bigcup_{i \in \omega} P_{2}^{i}\right)$, where:

(i) $P_{1}=(\text { Int })^{\omega} \subseteq\left(M_{C}\right)^{\omega}$, and

(ii) $P_{2}^{k}=\left\{\sigma \in\left(M_{C}\right)^{\omega}: \sigma(i) \in\right.$ Int for $i<k, \sigma(k) \in$ End, and for $i>k$, $\sigma(i) \in A_{j, k}$ where $\left.\sigma(k)=e_{j}\right\}$ for each $k \in \omega$.

We check that E1 satisfies the hypotheses of Theorem 1.4 of $\S$ III. It is obvious that $\left(M_{C}\right.$, Int, End) is a tame triple, so we only need to check that E1 respects $\left\{\left(M_{C}, \text { Int , End }\right)_{i}: i \in \omega\right\}$. From the definition of E1, condition (i) is satisfied. Letting $\phi_{1}: \omega \rightarrow \omega$ be defined by $\phi_{1}(i)=i+1$, and $\phi_{2}: \omega^{2} \rightarrow \omega$ be defined by $\phi_{2}(i, j)=\max \{i, j\}+1$, conditions (ii) and (iii) are met. Hence E1 is a zero-dimensional metrizable space, with $\operatorname{Ind}(\mathrm{E} 1) \leq 1$. In order to show $\mathrm{E} 1$ is complete, by Theorem 1.6 of $\S I$ we only need to check that it is approximately complete. To this end, suppose $\sigma \in\left(M_{C}\right)^{\omega}$ such that for all $i \in \omega$ there is $\sigma_{i} \in \mathrm{E} 1$ with $\left.\sigma_{i}\right|_{i}=\left.\sigma\right|_{i}$. If no $\sigma(j) \in \mathrm{End}$, then $\sigma \in P_{1}$, so suppose $\sigma(t) \in$ End. Say $\sigma(t)=e_{r}$. Then for each $i>t, \sigma_{i}$ must be in $P_{2}^{t}$, so 
$\sigma(j) \in$ Int for $j<t, \sigma(t)=\sigma_{i}(t)=e_{r}$, and $\sigma_{i}(j) \in A_{r, t}$ for $t<j<i$. It follows that $\sigma \in P_{2}^{t}$. Thus E1 is completely metrizable. That $\operatorname{Ind}(\mathrm{E} 1)>0$ will be shown in subsection 3 of this section; and $\mathbf{N}$-compactness is shown in subsection 4.

The following simpler modification of $\mathrm{E} 1$ can also be shown to have all of the important properties of El. Let $M$ denote the Michael line, $\mathbf{Q}$ the rational points, and $\mathbf{I}$ the irrational points. Let $\left\{A_{i}: i \in \mathbf{Q}\right\}$ partition $\mathbf{I}$ into second category everywhere sets.

Then $\mathrm{E}_{1}^{\prime}=R_{1} \cup\left(\bigcup_{i \in \omega} R_{2}^{i}\right)$, where

(i) $R_{1}=\mathbf{I}^{\omega} \subseteq M^{\omega}$, and

(ii) $R_{2}^{k}=\left\{\sigma \in M^{\omega}: \sigma(i) \in I\right.$ for $i<k, \sigma(k) \in \mathbf{Q}$, and for $i>k$, $\left.\sigma(i) \in A_{\sigma(k)}\right\}$ for $k \in \omega$.

We remark that $\mathrm{E} 1$ and $\mathrm{E} 1{ }^{\prime}$ have first countable zero-dimensional compactifications. To see this observe that $M_{C}$ and $M$ embed, in a natural way, in the Alexandroff duplicate, $A(C)$, of $C$. Thus $\mathrm{E} 1$ and $\mathrm{E} 1^{\prime}$ embed in $A(C)^{\omega}$.

2. Example E2. In order to get a subspace of $\Delta, \mathrm{E} 1$ must be changed so that factors are locally compact and locally countable, yet closures of subsets of $A$ are still "sufficiently large" in each factor.

Let $P=\{B\} \cup\left\{A_{b, k}: b \in B, K \in \omega\right\}$ be a partition of $C$ into Bernstein sets. Let $A=C \backslash B$, and let $S(B)=\left\{\left(s_{1}, s_{2}\right): s_{1}, s_{2}\right.$ are countably infinite subsets of $A$ with $\left.\left|\operatorname{cl}\left(s_{1}\right) \cap \mathrm{cl}\left(s_{2}\right)\right|=\mathfrak{c}\right\}$. A simple transfinite induction argument yields:

2.1. Lemma. There is a bijection $f: B \rightarrow S(B)$ satisfying: if $f(b)=\left(s_{1}, s_{2}\right)$ then $\left|J_{n}(b) \cap s_{1}\right|=\left|J_{n}(b) \cap s_{2}\right|=\omega$ for all $n \in \omega$.

Use this lemma to get $f$; let $f(b)$ be denoted by $\left(f_{1}(b), f_{2}(b)\right)$. A sequence $\tau_{b}$ can be chosen so that, for $i \in \omega$ :

(i) $\tau_{b}(2 i+1) \in f_{1}(b)$,

(ii) $\tau_{b}(2 i) \in f_{2}(b)$, and

(iii) $\tau_{b}(i) \in J_{i}(b)$.

Let $\tau_{b}^{n}=\left\{\tau_{b}(m): m \geq n\right\}$, and let $A_{k}=\bigcup_{b \in B} A_{b, k}$. Finally let $C^{\prime}$ denote the topological space with point set $C$, and a basis

$$
\{\{a\}: a \in A\} \cup\left\{\{b\} \cup \tau_{b}^{n}: b \in B, n \in \omega\right\} .
$$

It is clear that $C^{\prime}$ is a locally countable, locally compact, first countable refinement of $C$ and that $\left(C^{\prime}, A, B\right)$ is a tame triple, in the van Douwen line tradition.

Example E2 $=P_{1} \cup\left(\bigcup_{i \in \omega} P_{2}^{i}\right)$, where:

(i) $P_{1}=A^{\omega} \subseteq\left(C^{\prime}\right)^{\omega}$, and

(ii) $P_{2}^{k}=\left\{\sigma \in\left(C^{\prime}\right)^{\omega}: \sigma(i) \in A\right.$ for $i<k, \sigma(k) \in B$, and for $i>k$, $\sigma(i) \in A_{b, k}$ where $\left.\sigma(k)=b\right\}$ for $k \in \omega$. 
Showing E2 satisfies the hypotheses of Theorems 1.4 and 1.6 of $\S I I I$ is much like the proof for E1; we conclude that E2 is a zero-dimensional, completely metrizable space and that $\operatorname{Ind}(\mathrm{E} 2) \leq 1$.

3. In this subsection we show that $\operatorname{Ind}(\mathrm{E} 1)$ and Ind(E2) are positive. The technique here is based on Mrowka's modification of Roy's method. We prove a theorem which is general enough to show that a class of spaces containing E1 and E2 have positive Ind. There is an analogy here with $\S I I I$; there we wanted the subspace of the product to be small enough to be metrizable; here we want it large enough to have positive dimension.

3.1. Definition. A topology $T$ for $C$ is an acceptable refinement of $C$ provided there are subsets $A$ and $B$ of $C$ satisfying:

(i) $((C, T), A, B)$ is a tame triple,

(ii) $A$ is second category everywhere on $C$,

(iii) $(C, T)$ is a refinement of $C$, and

(iv) if $O$ is open in $C$, and $S_{1}, S_{2}$ are subsets of $A$ which are both second category everywhere on $O$, then there is $b \in B$ with $b \in \operatorname{cl}_{(C, T)}\left(S_{1}\right) \cap$ $\mathrm{cl}_{(C, T)}\left(S_{2}\right)$.

3.2. Definition. Let $\left\{T_{i}: i \in \omega\right\}$ be a collection of acceptable refinements of $C$, with sets $A_{i}, B_{i}$ corresponding to sets $A$ and $B$ in Definition 3.1. A subspace $K$ of $\prod_{i \in \omega}\left(C, T_{i}\right)$ is a full enough subspace of $\prod_{i \in \omega}\left(C, T_{i}\right)$ provided:

(i) $\prod_{i \in \omega} A_{i} \subseteq K$ and,

(ii) if $a_{i} \in A_{i}$ for $i<n$ and $b_{n} \in B_{n}$, then there are sets $A_{j}^{\prime} \subseteq A_{j}$ for each $j>n$ such that $A_{j}^{\prime}$ is second category everywhere on $C$ and $\left\langle\left(a_{0}, a_{1}, \ldots, a_{n-1}, b_{n}\right) ; D\left(a_{0}, a_{1}, \ldots, a_{n-1}, b_{n}\right)\right\rangle \subseteq K$ where $D\left(a_{0}, a_{1}, \ldots, a_{n-1}, b_{n}\right)=\prod_{n<j<\omega} A_{j}^{\prime}$.

We remark that both E1 and E2 are full enough subspaces of products of acceptable refinements of $C$. Thus the following theorem gives that $\operatorname{Ind}(\mathrm{E} 1)=$ $\operatorname{Ind}(\mathrm{E} 2)=1$.

3.3. Theorem. Let $\left\{T_{i}: i \in \omega\right\}$ be a collection of acceptable refinements of $C$, with $\left(\left(C, T_{i}\right), A_{i}, B_{i}\right)$ denoting the appropriate tame triples, and let $K$ be a full enough subspace of $\prod_{i \in \omega}\left(C, T_{i}\right)$ which respects $\left\{\left(\left(C, T_{i}\right), A_{i}, B_{i}\right): i \in \omega\right\}$. Then

(i) $K$ is a zero-dimensional metrizable space, and

(ii) $\operatorname{Ind}(K)=1$.

Since $K$ is respectful we need only show that $\operatorname{Ind}(K)>0$, since Theorem 1.4 of $\S$ III applies.

3.4. Proof of Theorem 3.3. We assume for all $i \in \omega$ and $b \in B_{i}$, a nested local base $\left\{N_{n}^{i}(b): n \in \omega\right\}$ in $\left(C, T_{i}\right)$ (as in 2.1 of $\S I I I$ ), with the further restriction that $N_{n}^{i}(b) \subseteq J_{n}(b)$; we also assume neighborhoods $\left\{V_{i}(\sigma): i \in \omega, \sigma \in k\right\}$ as 
in Definition 2.2 of $\S \mathrm{III}$, and that $K=P_{1} \cup P_{2}^{0} \cup P_{2}^{1} \cup \cdots$ as in Definition 1.3 of $\S$ III.

We now prove several lemmas which culminate in producing two closed subsets of $K$ with no partition of $K$ between them.

3.4.1. Lemma. Suppose the following are given.

(a) $k, s \in \omega, s \geq 1$,

(b) $a_{i} \in A_{i}$ for $i<k$,

(c) $b_{k} \in B_{k}$,

(d) open sets $O_{1}, O_{2}, \ldots, O_{s}$ in $C$ with $b_{k} \in O_{1}$, and

(e) $\left\{U_{1}, U_{2}\right\}$ an open cover of $K$.

Then there are

$\left(\mathrm{a}^{\prime}\right) i \in\{1,2\}$,

$\left(\mathrm{b}^{\prime}\right) s^{\prime} \geq s$,

( $\left.\mathrm{c}^{\prime}\right)$ open sets $O_{1}^{\prime}, O_{2}^{\prime}, \ldots, O_{s^{\prime}}^{\prime}$ in $C$, and

$\left(\mathrm{d}^{\prime}\right)$ a subset $D$ of $\left.D\left(a_{0}, a_{1}, \ldots, a_{k-1}, b_{k}\right)\right|_{s^{\prime}}$

which is super $S$-full on $\prod_{j=1}^{s^{\prime}}\left(O_{j}^{\prime} \cap A_{k+j}\right)$ and which satisfies:

(i) $O_{j}^{\prime} \subseteq O_{j+1}$ for $1 \leq j<s$, and

(ii) if $\sigma \in \overline{\left\langle\left(a_{0}, \ldots, a_{k-1}, b_{k}\right) ; D\right\rangle}$, then $V_{k+s^{\prime}}(\sigma) \subseteq U_{i}$.

Proof. Let $\hat{D}$ denote the set $\left\{d \in D\left(a_{0}, a_{1}, \ldots, a_{k-1}, b_{k}\right): d(i) \in O_{i+2}\right.$ for $i \leq s-2\}$. (We view $\hat{D}$ as a collection of sequences.) For each $d \in \hat{D}$, let $\sigma_{d}=\left\langle\left(a_{0}, \ldots, a_{k-1}, b_{k}\right) ; d\right\rangle \in K$. For $m \in \omega$, let $S_{m}=\left\{d \in \hat{D}: V_{k+m}\left(\sigma_{d}\right) \subseteq\right.$ $U_{1}$ or $\left.U_{2}\right\}$. Then it is easy to check that $\left\{S_{m}: m \in \omega\right\}$ satisfies the conditions of Lemma 4 of $\S I$, so there is $s^{\prime}$ such that $S_{s^{\prime}}$ contains $\hat{S}_{s^{\prime}}$ where $\left.\hat{S}_{s^{\prime}}\right|_{s^{\prime}}$ is an $S$-full subset of $\prod_{k<j \leq k+s^{\prime}}\left(C, T_{j}\right)$. Furthermore, if $\left.e \in \hat{S}_{s^{\prime}}\right|_{s^{\prime}}$ and $\sigma \in$ $\overline{\left\langle\left(a_{0}, a_{1}, \ldots, a_{k-1}, b_{k}\right) ; e\right\rangle}$, then $V_{k+s^{\prime}}(\sigma) \subseteq U_{1}$ or $U_{2}$. We may assume $s^{\prime} \geq$ $s$.

By the conditions on $\hat{D},\left.\hat{S}_{s^{\prime}}\right|_{s^{\prime}}$ is $S$-full on $\prod_{1<j \leq s} O_{j} \times \prod_{k+s \leq j \leq k+s^{\prime}}\left(C, T_{j}\right)$. Let $H_{i}=\left\{e \in \hat{S}_{s^{\prime}}: V_{k+s^{\prime}}\left(\sigma_{e}\right) \subset U_{i}\right\}$ for $i=1,2$. Then $H_{1} \cup H_{2}=\hat{S}_{s^{\prime}}$, and by Lemma 3 of $\S I I$, there is $i \in\{1,2\}$ such that $\left.H_{i}\right|_{s^{\prime}}$ contains a subset $D^{\prime}$ of $\left.\hat{S}_{s^{\prime}}\right|_{s^{\prime}}$ which is $S$-full on $\prod_{1<j \leq s} O_{j} \times \prod_{k+s \leq j \leq k+s^{\prime}}\left(C, T_{j}\right)$. By applying Lemma 5 of $\S$ II we get $O_{1}^{\prime}, O_{2}^{\prime}, \ldots, O_{s^{\prime}}^{\prime}$, and $D \subset D^{\prime}$.

3.4.2. Lemma. Suppose the following are given:

(a) $k, s \in \omega, s \geq 1$,

(b) $a_{j} \in A_{j}$ for $j<k$,

(c) $O_{1}, O_{2}, \ldots, O_{s}$ open sets in $C$,

(d) $\left\{U_{1}, U_{2}\right\}$ an open cover of $K$, and

(e) $D_{1}, D_{2}$ super $S$-full subsets of $\prod_{1 \leq j \leq s}\left(O_{j} \cap A_{k+j-1}\right)$ satisfying, for $i \in$ $\{1,2\}, \overline{\left\langle\left(a_{0}, \ldots, a_{k-1}\right) ; D_{i}\right\rangle} \subseteq U_{i}$. 
Then there are

$\left(\mathbf{a}^{\prime}\right) s^{\prime} \geq s$,

(b') $a_{k} \in A_{k}$,

(c' ${ }^{\prime} O_{1}^{\prime}, \ldots, O_{s^{\prime}}^{\prime}$, open sets in $C$, and

(d') $D_{1}^{\prime}, \quad D_{2}^{\prime}$ super $S$-full subsets of $\prod_{1 \leq j \leq s^{\prime}}\left(O_{j}^{\prime} \cap A_{k+j}\right)$ satisfying, for $i \in$ $\{1,2\}, \overline{\left\langle\left(a_{0}, \ldots, a_{k-1}, a_{k}\right) ; D_{i}^{\prime}\right\rangle} \subset U_{i}$.

Proof. For $j \in\{1,2\}$, let $F_{j}=\left\{d(0): d \in D_{j}\right\}$. Then $F_{1}, F_{2}$ are both second category everywhere on $O_{1}$, thus, from the fact that $T_{k}$ is an acceptable refinement of $C$, there is $b_{k} \in B_{k}$ with $b_{k} \in \operatorname{cl}_{\left(C, T_{k}\right)}\left(F_{1}\right) \cap \mathrm{cl}_{\left(C, T_{k}\right)}\left(F_{2}\right)$. Clearly $b_{k}$ can be chosen to be in $O_{1}$. Using $k, s$, the $O_{i}$ 's, $b_{k}$, and $\left\{U_{1}, U_{2}\right\}$, we can apply the previous lemma to get

(i) $i \in\{1,2\}$,

(ii) $s^{\prime} \geq s$,

(iii) open sets $O_{1}^{\prime}, O_{2}^{\prime}, \ldots, O_{s}^{\prime}$, in $C$, and

(iv) a subset $D_{i}^{\prime}$ of $\left.D\left(a_{0}, \ldots, a_{k-1}, b_{k}\right)\right|_{s^{\prime}}$ which is super $S$-full on $\prod_{1 \leq j \leq s^{\prime}}\left(O_{j}^{\prime} \cap A_{k+j}\right)$, and such that $O_{j}^{\prime} \subseteq O_{j+1}$ for $1 \leq j \leq s-1$ and for $\sigma \in \overline{\left\langle\left(a_{0}, \ldots, a_{k-1}, b_{k}\right) ; D_{i}^{\prime}\right\rangle}, V_{k+s^{\prime}}(\sigma) \subseteq U_{i}$.

Now, in $\left(C, T_{k}\right)$, if $a \in A_{k} \cap N_{k+s^{\prime}}^{k}\left(b_{k}\right)$, then $\overline{\left\langle\left(a_{0}, a_{1}, \ldots, a_{k-1}, a\right) ; D_{i}^{\prime}\right\rangle} \subseteq$ $U_{i}$. We may choose $a_{k} \in A_{k} \cap N_{k+s^{\prime}}^{k}\left(b_{k}\right)$ to be in $F_{2}$ if $i=1$ or $F_{1}$ if $i=2$. Suppose $i=1, a_{k} \in F_{2}$. Then $D_{1}^{\prime}$ is as required (using the $O_{i}^{\prime}$ 's).

To find $D_{2}^{\prime}$, first let $\hat{D}_{2}=\left\{\sigma \in \prod_{k \leq j<\omega} A_{j}:\left.\sigma\right|_{s} \in D_{2}, \sigma(0)=a_{k}\right.$, and $\sigma(i) \in O_{i}^{\prime}$ for $\left.1 \leq i \leq s^{\prime}\right\}$; let $\hat{\hat{D}}_{2}=\left\{(\sigma(1), \sigma(2), \ldots): \sigma \in \hat{D}_{2}\right\}$ and finally let $D_{2}^{\prime}=\left.\hat{D}_{2}\right|_{s^{\prime}}$. The super $S$-fullness on $D_{2}^{\prime}$ follows from the super $S$-fullness of $D_{2}$ and the fact that all $A_{n}$ 's are second category everywhere.

3.4.3. Lemma. Let $n \in \omega$ and let $F_{1}, F_{2}$ be subsets of $\prod_{i<n} A_{i}$ such that there are two distinct integers $n_{1}$ and $n_{2}$ with $\pi_{n_{i}}\left(F_{1}\right) \cap \pi_{n_{i}}\left(F_{2}\right)=\varnothing$ for $i \in\{1,2\}$. Then $\operatorname{cl}_{K}\left(\overline{\left\langle F_{1}\right\rangle}\right) \cap \mathrm{cl}_{K}\left(\overline{\left\langle F_{2}\right\rangle}\right)=\varnothing$.

Proof. If $\sigma \in P_{1}$, then either $\sigma\left(n_{1}\right) \notin \pi_{n_{1}}\left(F_{1}\right)$ or $\sigma\left(n_{1}\right) \notin \pi_{n_{1}}\left(F_{2}\right)$. Thus $V_{n_{1}+1}(\sigma)$ will miss one of $F_{1}$ and $F_{2}$. For $\sigma \in P_{2}^{k}$, then one of $n_{1}, n_{2} \neq k$, say $n_{1} \neq k$. It follows that $V_{n_{1}+1}(\sigma)$ misses either $F_{1}$ or $F_{2}$.

We now exhibit two closed sets in $K$ which permit no clopen partition of $K$ between them. For $i \in\{1,2\}$, partition $A_{i}$ into two sets $A_{i}^{1}$ and $A_{i}^{2}$ such that each of the partition elements is second category everywhere on $C$. Let $D_{i}=A_{i}^{1} \times A_{i}^{2}$ for $i \in\{1,2\}$. Choose $a_{0} \in A_{0}$ arbitrarily.

Let $F_{i}=\mathrm{cl}_{K}\left\langle\overline{\left(a_{0}\right) ; D_{i}}\right\rangle$. It is clear from the above lemma that $F_{1}$ and $F_{2}$ are nonintersecting closed sets in $K$.

3.4.4. There is no clopen partition of $K$ between $F_{1}$ and $F_{2}$, hence $\operatorname{Ind}(K)>$ 0 . 
Proof. Suppose $\left\{U_{1}, U_{2}\right\}$ is an open cover of $K$ with $F_{1} \subseteq U_{1}, F_{2} \subseteq U_{2}$. Using $k=1, s=2, O_{1}=O_{2}=C, a_{0}$, and $D_{1}, D_{2}$ as above, we can apply Lemma 3.4.3 to get $s^{\prime} \geq s, a_{1} \in A_{1}, O_{1}^{\prime}, \ldots, O_{s^{\prime}}^{\prime}$ open in $C$ and $D_{1}^{\prime}$, $D_{2}^{\prime}$ super $S$-full sets satisfying the conditions of 3.4.3. Lemma 3.4 .3 can be applied repeatedly in this fashion to produce $a_{2}, a_{3}, a_{4}, \ldots$ such that letting $\sigma=\left(a_{0}, a_{1}, a_{2}, \ldots\right)$, we have $\sigma \in P_{1}$, and each neighborhood of $\sigma$ intersects $U_{1}$ and $U_{2}$. Since $\left\{U_{1}, U_{2}\right\}$ covers $K, U_{1} \cap U_{2} \neq \varnothing$.

4. The purpose of this section is to show that spaces E1 and E2 are both $\mathbf{N}$ compact. The following result is easy and well known.

4.1. Lemma. If $\left(C^{\prime}, T\right)$ is zero-dimensional, where $C^{\prime} \subseteq C$ and $T$ gives a topology finer than the usual topology on $C^{\prime}$ as a subspace of $C$, then $\left(C^{\prime}, T\right)$ is hereditarily $\mathbf{N}$-compact.

The following corollary gives that both E1 and E2 are N-compact.

4.2. Corollary. Let $\left\{\left(C_{i}, T_{i}\right): i \in \omega\right\}$ be a collection of refinements of $C$; then $\prod_{i \in \omega}\left(C_{i}, T_{i}\right)$ is hereditarily $\mathbf{N}$-compact on zero-dimensional subspaces.

Proof. $\prod_{i \in \omega}\left(C_{i}, T_{i}\right)$ is a refinement of $C^{\omega}$ which is homeomorphic to $C$, thus Lemma 4.1 can be applied.

\section{A GAP METRIC SPACE OF WEIGHT $\mathfrak{c}$ WHOSE SUBSPACES OF WEIGHT SMALLER THAN $c$ ARE ALL STRONGLY ZERO-DIMENSIONAL}

A gap metric space is now described which contains no gap metric subspace of weight less than $\mathfrak{c}$. The factor spaces are refinements of the usual plane; in fact, any Euclidean space of dimension greater than or equal to two could be used in place of the plane, because we only make use of certain of its connectedness properties. It is interesting to compare this example with those in §II; here highly connected factor spaces allow the sets $A_{b, k}$ to be spread out in relation to each other, whereas in $\S \mathrm{II}$, in order to get $\mathbf{N}$-compactness, the factor spaces were highly disconnected and the sets $A_{b, k}$ then had to be extremely dense.

1.1. The factor space is a refinement of $R^{2}$ (the plane with the usual topology). Let $B$ be a Bernstein subset of $R^{2}$, and let $A=R^{2} \backslash B$. Then $R^{2 *}$ denotes the points set $R^{2}$ with topology generated by $B=\{\{\sigma\}: \sigma \in A\} \cup\left\{N_{n}(b): b \in B\right\}$ where $N_{n}(b)$ is the set of points whose distance to $b$ is less than $1 / n$. It is easy to verify that $\left(R^{2}, A, B\right)$ is a tame triple.

1.2. Example E3. Let $\left\{A_{i}: i \in \omega\right\}$ be a collection of subsets of $A$ such that:

(i) if $a_{i} \in A_{i}, a_{j} \in A_{j}$ and $i \neq j$, then the Euclidean distance between $a_{i}$ and $a_{j}$ is at least 1 , and

(ii) $\left|A_{i}\right|=\mathfrak{c}$ for all $i \in \omega$.

By (ii), each $A_{i}$ can be partitioned into $\left\{A_{b, i}: b \in B\right\}$ where each $A_{b, i}$ is uncountable. 
Example E3 is the subspace of $\left(R^{2 *}\right)^{\omega}$ given by E3 $=P_{1} \cup\left(\bigcup_{i \in \omega} P_{2}^{i}\right)$ where $P_{1}=A^{\omega}$, and for $k \in \omega, P_{2}^{k}=\left\{\sigma \in\left(R^{2 *}\right)^{\omega}: \sigma(i) \in A\right.$ for $i<k, \sigma(k) \in B$, and for $i>k, \sigma(i) \in A_{b, k}$ where $\left.\sigma(k)=b\right\}$.

Clearly E3 satisfies the hypotheses of Theorem 1.4 of $\S \mathrm{III}$, and so is metrizable, zero-dimensional, and $\operatorname{Ind}(\mathrm{E} 3) \leq 1$. It is also easy to check that E3 is complete using Theorem 1.6 of $\S I I I$.

1.3. Ind(E3) $\geq 1$. The proof of this follows the same general pattern as the proof in $\S I V$ for examples E1 and E2; in fact, in many ways it is easier. The following lemma is analogous to Lemma 3.4.1 of $\S \mathrm{IV}$, and the proof is omitted.

1.3.1. Lemma. Suppose $\left\{U_{1}, U_{2}\right\}$ is an open cover of E3, $k \in \omega$, for $j<k$, $a_{j} \in A$, and $b_{k} \in B$ are all given. Then there is an $m>0$, an $i \in\{1,2\}$ and a U-full subset $C$ of $\left(A_{b, k}\right)^{m}$ satisfying: If $\sigma \in \overline{\left\langle\left(a_{0}, \ldots, a_{k-1}, b_{k}\right) ; C\right\rangle}$, then $V_{k+m}(\sigma) \subseteq U_{i}$.

(Here $V_{t}(\sigma)$ is defined as in $\S$ III, 2.2, so that for $\sigma \in P_{1}, V_{t}(\sigma)=\{\tau \in$ E3: $\left.\left.\tau\right|_{t}=\left.\sigma\right|_{t}\right\}$ and for $\sigma \in P_{2}^{k}$ and $t>k, V_{t}(\sigma)=\left\{\tau \in \mathrm{E} 3:\left.\tau\right|_{k}=\left.\sigma\right|_{k}\right.$, $\tau(k) \in N_{t}(\sigma(k))$, and for $\left.k<j<t, \tau(j)=\sigma(j)\right\}$.)

The next lemma is analogous to Lemma 3.4.2 of $\S \mathrm{IV}$, but displays the use of the connectedness of the plane.

1.3.2. Lemma. Suppose $\left\{U_{1}, U_{2}\right\}$ is an open cover of E3, $k \in \omega$, and for $i<k, a_{i} \in A$ are given. Further suppose that for each $j \in\{1,2\}$ there is $m_{j}>0$ and $C_{j}$ a $U$-full subset of $A^{m_{j}}$ such that $\overline{\left\langle\left(a_{0}, \ldots, a_{k-1}\right) ; C_{j}\right\rangle} \subseteq U_{j}$. Then there is $a_{k} \in A$, and for $j \in\{1,2\}$ an $n_{j}>0$ and $U$-full sets $D_{j} \subseteq A^{n_{j}}$ such that

$$
\overline{\left\langle\left(a_{0}, \ldots, a_{k-1}, a_{k}\right) ; D_{j}\right\rangle} \subseteq U_{j} .
$$

Proof. For each $b \in B$, apply Lemma 1.3.1 to get $i_{b}, m_{b}$ and $C_{b}$ with $C_{b}$ a $U$-full set in $A^{m_{b}}$ satisfying: if $\sigma \in \overline{\left\langle\left(a_{0}, \ldots, a_{k-1}, b\right) ; C_{b}\right\rangle}$, then $V_{k+m_{b}}(\sigma) \subseteq$ $U_{i_{b}}$. Thus for $a \in A \cap N_{k+m_{b}}(b),\left\langle\left(a_{0}, \ldots, a_{k-1}, a\right) ; C_{b}\right\rangle \subset U_{i_{b}}$.

The collection $\left\{N_{k+m_{h}}(b): b \in B\right\}$ is an open cover of $B$ (in $R^{2}$ ), hence of all of $R^{2}$ except possibly a countable set. For $j \in\{1,2\}$, let $V_{j}=\left\{N_{k+m_{b}}(b)\right.$ : $\left.i_{b}=j\right\}$, and consider two cases.

Case 1. One of the $V_{j}$ 's is empty, say $V_{1}=\varnothing$. Because $C_{1}$ is $U$-full, $C_{1}^{\prime}=$ $\left\{c(0): c \in C_{1}\right\}$ is uncountable, and since $B$ is Bernstein, there is $b \in B$ with $N_{k+m_{b}}(b)$ containing $a \in C_{1}^{\prime}$. Let $a=a_{k}$ and let $n_{1}=1$. Then let $D_{1}=$ $\left\{\left(c(1), \ldots, c\left(m_{1}-1\right)\right): c \in C_{1}\right.$, and $\left.c(0)=a_{k}\right\}$. (If $m_{1}=1$, let $n_{1}=1$ and let $D_{1}$ be any uncountable subset of $A$.) Let $n_{2}=m_{b}$ and $D_{2}=C_{b}$.

Case 2. Both $V_{1}$ and $V_{2}$ are nonempty. Then there are for $j \in\{1,2\}, b_{j} \in B$ with $N_{k+m_{b_{j}}}\left(b_{j}\right) \in V_{j}$ and $N_{k+m_{b_{1}}}\left(b_{1}\right) \cap N_{k+m_{b_{2}}}\left(b_{2}\right) \neq \varnothing$. This is because no countable set separates the plane. Then $a_{k} \in N_{k+m_{b_{1}}}\left(b_{1}\right) \cap N_{k+m_{b_{1}}}\left(b_{2}\right) \cap A$ can 
be chosen, and setting $n_{j}=m_{b}$ and $D_{j}=C_{b}$ the conditions of the lemma are satisfied.

1.3.3. We describe the closed sets which exhibit Ind $>0$. Choose $a \in A$ and let $S_{1}, S_{2}$ be a pair of nonintersecting uncountable closed subsets of $R^{2}$ (so $\left|S_{i} \cap A\right|=\mathfrak{c}$ for $\left.i \in\{1,2\}\right)$. Then, setting $F_{i}=\overline{\left\langle(a) ; S_{i}\right\rangle}$ and repeatedly applying Lemma 1.3.2, it is shown that there is no clopen set containing $F_{1}$ and which is disjoint from $F_{2}$.

2. We now show that if $E 3^{\prime} \subset E 3$ and weight $\left(E 3^{\prime}\right)<\mathfrak{c}$, then $\operatorname{Ind}\left(E 3^{\prime}\right)=0$.

2.1. Lemma. If $\mathrm{E}^{\prime} \subset \mathrm{E} 3$, then for each $i \in \omega,\left|\pi_{i}\left(\mathrm{E}^{\prime}\right)\right| \leq$ weight(E3). Thus if weight $\left(E 3^{\prime}\right)<\mathfrak{c}, \pi_{i}\left(\mathrm{E}^{\prime}\right)$ is strongly zero-dimensional when viewed as a subset of $R^{2}$.

Proof. Let $\alpha=\left|\pi_{i}\left(\mathrm{E}^{\prime}\right)\right|$. We show $\alpha \leq$ weight $\left(\mathrm{E}^{\prime}\right)$ by showing that $\mathrm{E}^{\prime}$ contains $\alpha$ many pairwise disjoint distinct open sets. Suppose $\left|\pi_{i}\left(\mathrm{E}^{\prime}\right) \cap A\right|=\alpha$. Then for each $a \in\left(\pi_{i}\left(\mathrm{E}^{\prime}\right) \cap A\right)$ let $\sigma_{a}$ be a point of $P_{1} \cap \mathrm{E} 3^{\prime}$ such that $\sigma_{a}(i)=a$. It follows that $\left\{V_{i+1}\left(\sigma_{a}\right) \cap \mathrm{E}^{\prime}\right\}$ is a pairwise disjoint collection of nonempty open sets in $E 3^{\prime}$ of cardinality $\alpha$; hence $\alpha \leq$ weight $\left(E 3^{\prime}\right)$. Similarily, if $\left|\pi_{i}\left(\mathrm{E}^{\prime}\right) \cap B\right|=\alpha$, we can find $\tau_{b} \in P_{2}^{i} \cap \mathrm{E}^{\prime}$ for each $b \in\left(\pi_{i}\left(\mathrm{E}^{\prime}\right) \cap B\right)$, with $\tau_{b}(i)=b$. Then $\left\{V_{i+2}\left(\tau_{b}\right) \cap \mathrm{E} 3^{\prime}\right\}$ is the desired collection.

By this result we will be done when we show

2.2. Lemma. If $\mathrm{E}^{\prime} \subset \mathrm{E} 3$, and for each $i \in \omega, \pi_{i}\left(\mathrm{E}^{\prime}\right)$ is a zero-dimensional subset of $R^{2}$, then $\operatorname{Ind}\left(\mathrm{E}^{\prime}\right)=0$.

Proof. The goal is to construct a sequence $\left\{H_{i}: i \in \omega\right\}$ of covers of $\mathrm{E}^{\prime}$ such that for all $i \in \omega, H_{i+1}$ refines $H_{i}$, $\operatorname{order}\left(H_{i}\right)=1$ and $\bigcup_{i \in \omega} H_{i}$ forms a base for $E 3^{\prime}$. It will follow by [N, Theorem 12-6] that $\operatorname{Ind}\left(\mathrm{E3}^{\prime}\right)=0$.

First we get such covers in the factors. For each $i \in \omega$, let $\left\{G_{n}^{i}: n \in \omega\right\}$ be a collection of covers of $\pi_{i}\left(\mathrm{E}^{\prime}\right)$ (open in the $R^{2}$ topology) such that for each $n \in \omega, \operatorname{order}\left(G_{n}^{i}\right)=1, G_{n+1}^{i}$ refines $G_{n}^{i}$, and if $g \in G_{n}^{i}$ then the $\left(R^{2}\right)$ diameter of $g$ is less than $1 / n+1$. The $G_{n}^{i}$ 's can be obtained because $\pi_{i}\left(\mathrm{E3}^{\prime}\right)$ is a strongly zero-dimensional subset of $R^{2}$. Note that if $g \in \bigcup_{n \in \omega} G_{n}^{i}$ then $g$ can intersect no more than one element of $\left\{A_{k}: k \in \omega\right\}$, by our choice of $A_{i}$ 's. For $p \in \pi_{k}\left(\mathrm{E}^{\prime}\right)$, let $g_{n}^{k}(p)$ denote the element of $G_{n}^{k}$ which contains $p$. We may assume that if $\sigma \in P_{1}$, and $\left.\sigma\right|_{n}=\left.\tau\right|_{n}$ for some $\tau \in \mathrm{E}^{\prime}$, then there is $\gamma \in \mathrm{E}^{\prime} \cap P_{1}$ with $\left.\gamma\right|_{n}=\left.\sigma\right|_{n}$. Simply add the sequence $\delta$ with $\delta(i)=\sigma(i)$ for $i<n$, and $\delta(i)=0$ for $i>n$. (Make sure 0 is not in $B$.) We can also assume that if $\tau \in \mathrm{E}^{\prime} \cap P_{1}$, then $\left\{\gamma(n):\left.\gamma\right|_{n}=\left.\tau\right|_{n}\right\}$ is dense in $R^{2}$, by choosing a countable dense subset of $A \cap R^{2}$, and letting elements of that set "follow" $\left.\tau\right|_{n}$.

We now define a new base on E3'.

(i) For $\sigma \in \mathrm{E}^{\prime} \cap P_{1}$, and $m \in \omega, U_{m}(\sigma)=\left\{\tau \in \mathrm{E}^{\prime}:\left.\tau\right|_{m}=\left.\sigma\right|_{m}\right.$ and $\left.\tau(m) \in g_{m}^{m}(\sigma)\right\}$ 
(ii) For $k \in \omega, \sigma \in \mathrm{E} 3^{\prime} \cap P_{2}^{k}$, and $m>k$, let $U_{m}(\sigma)=\left\{\tau \in \mathrm{E} 3^{\prime}:\left.\tau\right|_{k}=\right.$ $\left.\sigma\right|_{k}, \tau(k) \in g_{m}^{k}(\sigma(k))$, for $k<i<m, \tau(i)=\sigma(i)$, and $\tau(m) \in$ $\left.g_{m}^{m}(\sigma(m))\right\}$.

It is easy to verify that $\left\{U_{m}(\sigma): \sigma \in \mathrm{E}^{\prime}, m \in \omega \quad\left(m>k\right.\right.$ if $\left.\left.\sigma \in P_{2}^{k}\right)\right\}$ forms a base for $\mathrm{E3}^{\prime}$.

Setting $H_{i}^{1}=\left\{U_{i}(\sigma): \sigma \in P_{1}\right\}$ and $H_{i}^{2}=\left\{U_{i}(\sigma): \sigma \in P_{2}^{0} \cup \cdots \cup P_{2}^{i-1}\right\}$, $H_{i}^{1} \cup H_{i}^{2}$ covers $\mathrm{E}^{\prime}$, and each is order one. If $h \in H_{i}^{1}$ and $k \in H_{i}^{2}$ intersect, then $h \subseteq k$. Let $H_{i}=H_{i}^{2} \cup\left\{h \in H_{i}^{1}: h\right.$ is not a subset of $\left.\cup H_{i}^{2}\right\}$. Then $\left\{H_{i}: i \in \omega\right\}$ satisfies the conditions laid out at the beginning of the proof.

2.3. Remark. By carefully choosing the sets $\left\{A_{b, i}: b \in B, i \in \omega\right\}$ and a subspace of the so-defined version of E3 an example which is both $\mathbf{N}$-compact and contains no subspace with weight less than $c$ of positive dimension can be found.

\section{CONSISTENT EXAMPLES OF METRIZABLE SPACES OF POSITIVE Ind FOR WHICH SMALL SUBSPACES HAVE Ind $=0$}

For $\lambda$ an ordinal, we consider $\lambda$ a topological space with the order topology; a subset of $\lambda$ is assumed to have the subspace topology. We let $\operatorname{Suc}(\lambda)$ and $\operatorname{Lim}(\lambda)$ respectively denote the sets of successors and limits in $\lambda$.

For a cardinal $\kappa, E(\kappa)$ is the statement: There is a set $E$ of ordinals of cofinality $\omega$ which is stationary in $\kappa$, but for all $\beta<\kappa, E \cap \beta$ is not stationary in $\beta$.

The following statement, which we call $E^{*}$, is consistent with the usual axioms of set theory.

$E^{*}$ : For all singular strong limit cardinals $\kappa, 2^{\kappa}=\kappa^{+}$and $E\left(\kappa^{+}\right)$.

For a discussion of $E^{*}$, the reader should consult [F]. The author would like to thank Bill Fleissner for pointing out $E^{*}$ and also for the following lemma.

1. Lemma. Let $\kappa$ be a cardinal for which $E(\kappa)$ holds, and let $E_{\kappa}$ denote the set guaranteed by $E(\kappa)$. Let $\delta<\kappa$ be fixed, and let $M_{\delta}=\delta \cap\left(\operatorname{Suc}(\kappa) \cup E_{\kappa}\right)$. Then $M_{\delta}$ is metrizable and $\operatorname{Ind}\left(M_{\delta}\right)=0$.

Proof. We first get an open cover $H$ of $M_{\delta}$ which is locally finite such that $H=\{\{\beta\}: \beta \in \operatorname{Suc}(\delta)\} \cup\left\{\left(\beta^{\prime}, \beta\right]: \beta \in E_{\kappa} \cap M_{\delta}\right.$ and $\beta^{\prime}$ depends on $\left.\beta\right\}$. To get this cover, proceed by transfinite induction; assume for all $\alpha<\delta$ the cover exists, and $\delta<\kappa$. Then there is a $C$ which is closed and unbounded in $\delta$ and disjoint from $E_{\kappa}$. Let $D_{c}=\left\{c_{\beta}: \beta<\operatorname{cof}(\delta)\right\}$ be a relatively discrete, cofinal in $\delta$ subset of $C$. For $c_{\beta} \in D_{c}$, let $s_{\beta}=\sup \left\{c_{\gamma}: \gamma<\beta\right\}$. By the inductive hypothesis there is a cover $H_{\beta}^{\prime}$ of $\left[0, c_{\beta}\right] \cap M_{\delta}$ as specified. Let $H_{\beta}=\left\{h \cap\left(s_{\beta}, c_{\beta}\right] \cap M_{\delta}: h \in H_{\beta}^{\prime}\right\}$, and let $H=\bigcup_{\beta<\operatorname{cof}(\delta)} H_{\beta}$. Now for $\beta \in$ $E_{\kappa} \cap M_{\delta}$ let $\left\{\beta_{i}: i \in \omega\right\}$ be an increasing sequence in $\delta$ converging to $\beta$ such 
that $\left(\beta_{0}, \beta\right] \subseteq\left(\beta^{\prime}, \beta\right]$ where $M_{\dot{\delta}} \cap\left(\beta^{\prime}, \beta\right] \in H$. For $i \in \omega$, let

$$
H_{i}=\{\{\beta\}: \beta \in \operatorname{Suc}(\delta)\} \cup\left\{\left(\beta_{i}, \beta\right] \cap M_{\delta}: \beta \in E_{\kappa} \cap \delta\right\} .
$$

One easily checks that $H_{i+1}$ refines $H_{i}, \bigcup_{i \in \omega} H_{i}$ is a base for $M_{\delta}$, and $H_{i}$ is a locally finite collection of clopen sets. Thus $M_{\delta}$ is a metrizable space and $\operatorname{Ind}\left(M_{\delta}\right)=0$.

Immediately from this lemma we get

2. Lemma. If $\kappa$ is a regular cardinal and $E(\kappa)$ holds, with $E_{\kappa}$ the set guaranteed by $E(\kappa)$, and $\delta<\kappa$, then if $X \subseteq\left(\operatorname{Suc}(\kappa) \cup E_{\kappa}\right)^{\omega}$ and weight $(X) \leq \delta$, then $X$ is metrizable and $\operatorname{Ind}(X)=0$.

Proof. Let $\alpha=\sup \left\{\pi_{k}(X): k \in \omega\right\}$. Then $\alpha<\kappa$, and

$$
X \subseteq\left(\left(\operatorname{Suc}(\kappa) \cup E_{\kappa}\right) \cap \alpha\right)^{\omega},
$$

and by the previous lemma $\left(\left(\operatorname{Suc}(\kappa) \cup E_{\kappa}\right) \cap \alpha\right)$ is metrizable and has Ind = 0 . Thus $\left(\left(\operatorname{Suc}(\kappa) \cup E_{\kappa}\right) \cap \alpha\right)^{\omega}$ is metrizable with Ind $=0$, and hence $X$ is metrizable with Ind $=0$.

3. Theorem (Assume $E^{*}$ ). For each cardinal $\lambda$ there is a metrizable space $X(\lambda)$ with $\operatorname{Ind}(X(\lambda))=1$ and for all $Y \subseteq X(\lambda)$, with weight $(Y) \leq \lambda, \operatorname{Ind}(Y)=0$.

Proof. Our proof is patterned after that in [K1]; we intend to replace the set $\omega_{1}$ with the set $\operatorname{Suc}(\kappa) \cup E(\kappa)$ for a sufficiently large $\kappa$.

Let $\kappa$ be a strong limit cardinal greater than $\lambda$; then $\kappa^{+}$is a regular cardinal, and $E^{*}$ gives that $E\left(\kappa^{+}\right)$holds. Let $E_{\kappa^{+}}$denote the set it gives.

Our example is a subspace of $W^{\omega}$ where $W=\operatorname{Suc}\left(\kappa^{+}\right) \cup E\left(\kappa^{+}\right)$. Observe that by the previous lemma, any subspace of $W^{\omega}$ of weight $\leq \lambda$ is necessarily metrizable with Ind $=0$. So we need only produce a positive dimensional subspace of $W^{\omega}$. Since $\left(W, \operatorname{Suc}\left(\kappa^{+}\right), E\left(\kappa^{+}\right)\right)$is a tame triple we want to satisfy the other hypothesis of Theorem 1.4 of $\S$ III. We define $X(\lambda) \subseteq W^{\omega}$ by $X(\lambda)=P_{1} \cup\left(\bigcup_{i \in \omega} P_{2}^{i}\right)$ where:

(i) $P_{1}=\left(\operatorname{Suc}\left(\kappa^{+}\right)\right)^{\omega}$ and for $k \in \omega$,

(ii) $P_{2}^{k}=\bigcup_{r \in E_{\kappa}}\left\{\sigma \in W^{\omega}: \sigma(i) \in \operatorname{Suc}\left(\kappa^{+}\right)\right.$for $i<k, \sigma(k)=\alpha, \sigma(k+1)=$ $\alpha+k$, and for $\left.i>k+1, \sigma(i) \in\left\{e+k: e \in \operatorname{Lim}\left(\kappa^{+}\right)\right\}\right\}$.

One can check, using $\phi_{1}(i)=i+1$ and $\phi_{2}(i, j)=\max \{i, j\}+1$, that the hypotheses of Theorem 1.4 of $\S$ III are satisfied; also one can show $X(\lambda)$ is complete using Theorem 1.6 of $\S I I I$. Proving that $\operatorname{Ind}(X(\lambda))>0$ is very similar to the proof that $\operatorname{Ind}(Z)>0$ in [K1], the main difference is in substituting $U\left(\kappa^{+}\right)$-full for $U$-full, where a set $S$ has property $U\left(\kappa^{+}\right)$iff $|S|=\kappa^{+}$. Also, it can be shown that if $F_{1}, F_{2}$ are disjoint closed subsets of $X(\lambda)$ and the weight of $F_{1}$ is less than or equal to $\lambda$, then there is a clopen set containing $F_{1}$ and missing $F_{2}$. This can be done using the methods in $\S 5$ of [K1]. 


\section{REFERENCES}

[A] C. E. Aull, Problem section, Lecture Notes in Pure and Appl. Math., vol. 95, Marcel Dekker, New York and Basel, 1985, pp. 311-314.

[F] W. G. Fleissner, The normal Moore space conjecture and large cardinals, Handbook of Set Theoretic Topology, North-Holland, Amsterdam, 1984, pp. 733-760.

[K1] J. Kulesza, An example in the dimension theory of metrizable spaces, Topology Appl. (to appear).

[K2] Dissertation, SUNY at Binghamton, 1987.

[M] S. Mrowka, N-compactness, metrizability, and covering dimension, Lecture Notes in Pure and Appl. Math., vol. 95, Marcel Dekker, New York and Basel, 1985, pp. 247-275.

[N] G. Naber, Set theoretic topology, University Microfilms International, Ann Arbor, Mich., 1977.

[NA] K. Nagami, Dimension theory, Academic Press, New York, 1970.

[NY] P. J. Nyikos, Prabir Roy's space $\Delta$ is not N-compact, General Topology Appl. 3 (1973), 197-210.

[O] A. Ostaszewski, $A$ note on the Prabir Roy space $\Delta$, preprint.

[R1] P. Roy, Failure of equivalence of dimension concepts for metric spaces, Bull. Amer. Math. Soc. 68 (1962), 609-613.

[R2] 117-132.

Department of Mathematical Sciences, George Mason University, fairfax, Virginia 22030 